\title{
Genetic monogamy and mate choice in a pair-living primate
}

\author{
Sofya Dolotovskaya ${ }^{1}$, Christian Roos ${ }^{2}$, and Eckhard Heymann ${ }^{3}$ \\ ${ }^{1}$ German Primate Centre Leibniz Institute for Primate Research \\ ${ }^{2}$ German Primate Centere \\ ${ }^{3}$ German Primate Center
}

August 28, 2020

\begin{abstract}
In pair-living mammals, genetic monogamy is extremely rare. One possible reason is that in socially monogamous animals, mate choice can be severely constrained, generating a risk of inbreeding or incompatibility between partners. To escape these constraints and minimize inbreeding, individuals might engage in extrapair copulations. Alternatively, inbreeding can be avoided by natal dispersal. However, little is known about the interactions between mating system, mate choice and dispersal in pair-living mammals. Here we show that coppery titi monkeys (Plecturocebus cupreus), are mostly genetically monogamous, as parentage analyses indicated no cases of extra-pair paternity. We did not find evidence for relatedness- or heterozygosity-based mate choice. Despite the lack of evidence for active inbreeding avoidance via mate choice, mating partners were on average not related. We further found that dispersal was opportunistic, with both sexes dispersing over varying distances. Our findings indicate that even opportunistic dispersal, as long as it is not constrained, can generate sufficient genetic diversity to prevent inbreeding. This, in turn, can render both active inbreeding avoidance via mate choice and extra-pair copulations unnecessary, thus helping to maintain genetic monogamy.
\end{abstract}

\section{Key words}

Genetic monogamy, mate choice, dispersal, titi monkeys, Plecturocebus

\section{Introduction}

Since sexual selection in both males and females is influenced by the number of mating partners, extrapair paternities (EPP) play an important role in the evolution of mating systems ${ }^{1,2}$. EPP are common in pair-living, or socially monogamous birds and mammals (see Table 1 for definitions used in this study), including humans, while genetic monogamy is a very rare phenomenon ${ }^{1,3,4}$. Among pair-living mammals which constitute up to $9 \%$ of mammal species, depending on the classification method ${ }^{5,6}$ - strict genetic monogamy (no cases of EPP) has been reported for only seven species so far (Table 2). Four other species can be considered as "mostly" genetically monogamous, with the rate of EPP $<10 \%$. However, for most pairliving mammal species, genetic paternity data simply does not exist yet, and therefore our understanding of the frequency of genetic monogamy is very incomplete.

Rates of EPP vary substantially between species and populations and have been shown to be affected by various factors, such as, for example, intensity of male care, pair-bond strength and population density $3,4,7,8$. The intriguing question is why some individuals engage in mating with multiple partners while others do not. The advantages to males of engaging in extra-pair copulations (EPC) are well recognized, as males are expected to increase their fitness by increasing the number of mating partners as the result of their higher potential reproductive rate ${ }^{2,9}$. However, in pair-living species with biparental care, potential reproductive 
rates and, consequently, levels of intra-sexual competition will be more similar for males and females ${ }^{2}$. As a result, both sexes might be expected to gain benefits from engaging in EPC ${ }^{10}$.

One potential advantage of EPC to females could to be related to limitations in mate choice. In pair-living species with biparental care, especially in those with low mobility and low breeding density, mate choice can be highly constrained. Not only do mates become unavailable once paired, but also individuals may face a conflict between choice for direct benefits (territory, paternal care) and indirect genetic benefits. As a result, individuals may end up paired to a genetically incompatible or to a closely related partner. To escape these constraints and minimize inbreeding, animals might seek EPC that would allow them to gain indirect benefits while still taking advantage of direct benefits provided by the social partner ${ }^{10}$. This strategy has been demonstrated in various bird species ${ }^{7,11}$. In mammals, the evidence is much more limited. In Alpine marmots, Marmota marmota, and meerkats, Suricata suricatta EPP rates were found to be higher in pairs where partners were more closely related ${ }^{12,13}$. But, to our knowledge, the only pair-living mammal species for which this effect has been demonstrated is the fat-tailed dwarf lemur, Cheirogaleus medius, where females sharing more major histocompatibility complex (MHC)-supertypes with their social partner engaged in more $\mathrm{EPC}^{14}$.

Whether an individual chooses to restrict matings to its social partner or to seek EPC, it is expected to do so to maximize not only its direct fitness benefits, but also the indirect (genetic) benefits, expressed as increased genetic quality of offspring. The closely related hypotheses of genetic compatibility and heterozygosity posit that individuals benefit from choosing a mate that will maximize offspring heterozygosity ${ }^{15-17}$. Thus, animals are expected to choose mates that are genetically unrelated or dissimilar at some fitness-related genes (e.g., MHC genes). An increase in offspring heterozygosity resulting from this disassortative mating is expected increase offspring fitness, as indicated by links between individual heterozygosity and various fitness proxies, such as survival, reproductive success and parasite resistance (e.g., Coltman et al., 1999; Foerster et al., 2003; Ortego et al., 2007) reviewed in Kempenaers (2007). In addition, irrespective of genetic compatibility, individuals might also benefit from choosing heterozygous mates, because heterozygous partners are expected to have higher fitness and should be more likely to provide direct benefits such as increased parental care, fertility or good quality territory ${ }^{15,20}$.

Mate choice based on heterozygosity was demonstrated in various species of birds and mammals. For example, in blue tits, Cyanistes caeruleus, heterozygosity was positively correlated between social mates, indicating that mating preferences were based on partner's heterozygosity ${ }^{21}$. In Antarctic fur seals, Arctocephalus gazella, where females exert choice by moving across a breeding colony to visit largely stationary males, females were shown to move further to optimize between high heterozygosity and low relatedness ${ }^{20}$. In many species, mate choice was shown to be based on MHC loci dissimilarity (e.g., fat-tailed dwarf lemur, Cheirogaleus medius: ${ }^{14}$ or, conversely, similarity (probably an adaptation to local pathogens, shown in, e.g., Malagasy giant jumping rat, Hypogeomys antimena, and European badgers, Meles meles ${ }^{22,23}$. Finally, relatedness-based mate choice, while demonstrated in some species, such as Antarctic fur seals, was not found in many other studied species, such as fat-tailed dwarf lemurs, blue titis and great reed warblers, Acrocephalus arundinaceus (García-Navas et al., 2009; Hansson et al., 2007; Schwensow et al., 2008; Sommer, 2005).

One of the biggest problems arising from the constraints of mate choice in pair-living animals is the risk of inbreeding. In the absence of other options, or as a result of a trade-off between choosing a good territory and unrelated/compatible partner, individuals might pair with too closely related mates. This problem can be solved "actively" by either avoiding matings with closely related individuals (through kin recognition) or engaging in EPC with less related individuals, as discussed above ${ }^{10,25}$. Alternatively, "passive" inbreeding avoidance can be ensured by natal dispersal that disrupts opposite-sex kin associations and thus allows to avoid matings between them ${ }^{26}$. Dispersal was shown to be sufficient to avoid inbreeding or reach a certain level of genetic dissimilarity in many situations ${ }^{21,24,27}$. However, it remains unclear if dispersal has to be sex-biased to generate enough local genetic dissimilarity between breeding females and males to avoid inbreeding. In most mammals, males are the dispersing sex, because in polygynous mating systems, which are prevailing in mammals, males experience stronger intra-sexual competition for mates than females 
26,28. Following the same logic, mammals that mate monogamously or cooperatively with high levels of reproductive monopolization by a dominant pair are expected to have little or no sex bias in dispersal. This was found to be true in some mammals, such as the genetically monogamous Azara's owl monkey, Aotus azarae, where both sexes disperse, or cooperatively breeding meerkats, where dispersal is only slightly male-biased ${ }^{29,30}$. However, in other mammals, e.g., genetically monogamous California mice, Peromyscus californicus, or socially monogamous greater white-toothed shrew, Crocidura russula, dispersal was found to be female-biased ${ }^{31,32}$.

Here, we present a comprehensive study of the genetic mating system, mate choice and dispersal in a wild population of coppery titi monkeys, Plecturocebus cupreus. Titi monkeys (genera Callicebus, Plecturocebus, and Cheracebus) exhibit almost all the elements of the "monogamy package", such as pair living, strong long-term pair bonds, an exceptionally high level of male care (the infant is carried almost exclusively by the social father), territoriality, and sexual monomorphism ${ }^{33-36}$. The only missing component which has yet to be characterized is the genetic mating system. Titis are one of the very few mammalian taxa that exhibit both high level of male care and strong pair bonds, two characteristics shown to affect the rates of EPP in mammals ${ }^{3}$. The examination of their mating system and the proximate mechanisms of its maintenance may, therefore, shed light on the evolution of social and genetic monogamy in mammals. In this study, we first examined the mating system of coppery titis using a set of 27 newly developed microsatellite loci that can be universally applied to New World monkeys. Second, we tested for evidence of relatedness- and/or heterozygosity-based mate choice. Finally, to see if dispersal is sex-biased, we compared genetic relatedness and diversity patterns in adult females and males and performed spatial genetic analysis. Given consistent pair living, strong pair bonds and high levels of male care in coppery titis, we predicted them to be genetically monogamous or have a very low rate of EPP. Since the risk of inbreeding is expected to be especially high for long-lived pair-living species such as titis, we expected to find evidence for active inbreeding avoidance via mate choice and/or for heterozygosity-based mate choice. We predicted both sexes to disperse, as expected from a pair-living territorial mammal with biparental care.

\section{Results}

Are titis genetically monogamous?

Our analyses did not indicate any cases of EPP. In all cases of assigned paternity (17 offspring in 9 social groups, 1 to 5 offspring per group; Fig. 1, Supplementary Table S1), social fathers were identified as genetic fathers of all offspring in their respective family groups. In one case, paternity remained unassigned. The juvenile offspring from Group 10 had three mismatches with the adult male of the group, and Delta score calculated by Cervus (the difference between the likelihood ratios for two most likely candidate parents) was zero, indicating both this male and the adult male of Group 8 as most likely fathers. At one of the loci with mismatches (chr09a), the offspring was homozygous, likely resulting from allelic dropout or genotyping error, at other two loci (chr07a, chr08a), the offspring was heterozygous, so we can only suggest that it was a result of a genotyping error. Unfortunately, this offspring had the minimum number of typed loci among all the sampled animals and also was the only individual in our dataset for whom we only had one fecal sample collected. Therefore, we could not control for the errors using another sample like we did for all other individuals. 


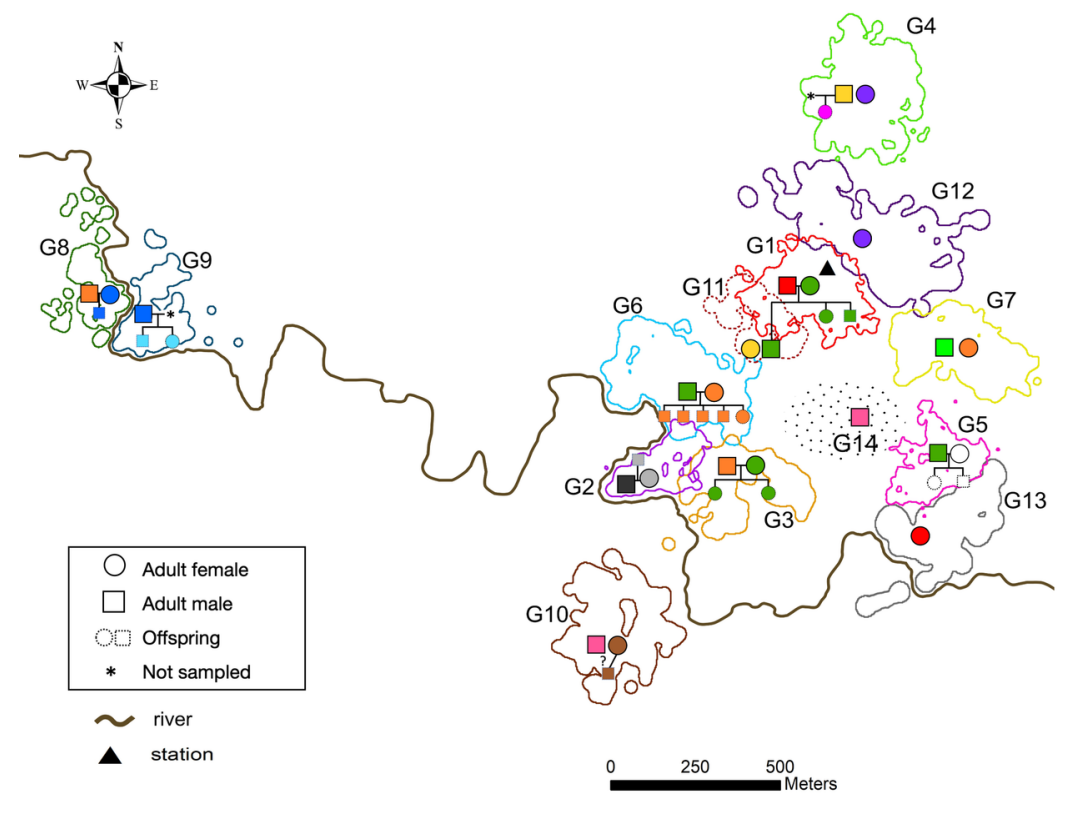

Figure 1: Home ranges, mtDNA haplotypes, and parentage for sampled individuals within study groups. Circles and squares with continuous outline represent adult females and males, respectively; smaller circles and squares with dotted outline represent female and male offspring, respectively. The colors of circles and squares represent different mtDNA haplotypes. Home ranges of study groups were estimated using the $95 \%$ fixed kernel density method with ArcGIS Desktop 10.6 (ESRI). The home range of Group 14 is depicted as dotted ellipse because we did not have enough GPS data to reliably estimate its home range. The home range of Group 11 is depicted as dotted line because this newly established territory was most likely not permanent and bound to shift later.

Apart from the three mismatches in the case of the unassigned paternity, we found only two cases of allelic mismatches (Supplementary Table S1). Father-daughter dyad from Group 1 had a mismatch at locus chr10a. Since the daughter was homozygous at this locus, this was most likely a result of allelic dropout or genotyping error. Father-daughter dyad from Group 9 had a mismatch at locus chrXa; the daughter was not homozygous at this locus but considering the high likelihood of parentage given by the other loci, we assumed that this mismatch was due to a genotyping error.

Of eight sampled social mothers, seven were identified as genetic mothers of all offspring in their family groups (17 offspring in 7 groups, 1 to 5 offspring per group). One inferred case of female replacement was detected, as the adult female of Group 4 was not identified as the genetic mother of the group's juvenile offspring; they did not share the mtDNA haplotype and had 11 allelic mismatches. No other female in our sample was identified as the most likely mother for this offspring or shared a mtDNA haplotype with it. The social father of this juvenile was indicated as the genetic father.

All assignments were made with a $95 \%$ confidence level in Cervus software and confirmed with Colony software (Supplementary Table S1). The assignments did not change when the set of known mother-offspring pairs was excluded from the priors. Colony also yielded strong support for full-sib relationships between all offspring from the same groups, confirming correct parentage assignments.

\section{Is mate choice based on relatedness or heterozygosity?}

We found no evidence for relatedness-based mate choice. There was no difference between relatedness of real mating pairs and randomly generated mating pairs (-0.048 vs. $-0.021, \mathrm{p}=0.565 ; \mathrm{n}=10$ pairs, breeding pool of 12 females and 12 males). Likewise, we found no evidence for heterozygosity-based mate choice, as 
homozygosity by loci (HL) was not significantly correlated between pair mates $(\mathrm{r}=-0.527, \mathrm{n}=10$ pairs, $\mathrm{p}$ $=0.118)$.

Despite the lack of evidence for active inbreeding avoidance via mate choice, relatedness (Wang's $r$ ) between mating partners was generally low, averaging -0.033 , and none of the pair mates shared the same mtDNA haplotype (Supplementary Table 1, Fig. 1). Only in one pair were the partners found to be second-degree kin (Group 6, $r=0.285$ ). The mtDNA haplotype network (Fig. 2) showed no clear pattern of haplotype similarity between pair mates: some had closely related haplotypes (e.g., Groups 4, 5, 9), while others had only distantly related haplotypes (e.g., Groups 1, 11).

Do both sexes disperse and does one sex disperse further than the other?

Our results indicate that both sexes dispersed similar distances. There were no significant differences between adult females and males in mean mtDNA haplotype diversity ( 0.945 in females, 0.924 in males, permutation test $\mathrm{p}=0.766)$, mtDNA nucleotide diversity $(0.027$ in females, 0.029 in males, permutation test $\mathrm{p}=0.699)$, mean relatedness $r$ (-0.013 in females, -0.056 in males, mean difference -0.040 , lying within the $95 \%$ confidence interval (-0.048 - 0.054) obtained by bootstrapping) or mean heterozygosity HL (0.184 in females, 0.216 in males, paired t-test $\mathrm{p}=0.438)$.

We did not find evidence for spatial genetic structure in our study population, suggesting that dispersal is most likely opportunistic. The correlation between genetic and spatial distances was not significant for either sex, as the $95 \%$ CI of autocorrelation $\mathrm{r}$ values overlapped zero for all distance classes (Supplementary Materials Table 2, Fig. S1). The correlation between mtDNA haplotype distances and spatial distances in females was not significant either (Mantel correlation $=0.048, \mathrm{n}=91$ dyads, right-tailed $\mathrm{p}=0.342$ ).

\section{Discussion}

The link between mating system, mate choice and dispersal has rarely been studied in pair-living mammals. Here, we demonstrated that coppery titi monkeys are mostly genetic monogamous, as we did not find evidence for EPP in our study population. We also did not find evidence for relatedness- or heterozygosity-based mate choice. Despite the absence of evidence for active inbreeding avoidance via mate choice, pair mates in our study population had low average relatedness. This finding suggests that in our study population, natal dispersal generates sufficient level of genetic dissimilarity between females and males to render both active inbreeding avoidance and EPC unnecessary.

Coppery titis are only the second primate species and the seventh pair-living mammal with no evidence of EPP found in a study with an adequate sample size (the study on Bornean gibbon was based on just four infants from four family groups ${ }^{37}$, Table 1 ). The absence of EPP in titis is not unexpected, as they are consistently pair-living, pair mates spend most of the day within a few meters from each other, sleep together at night and engage in frequent joint visual displays and duetting at the territorial borders $36,38-41$. This high level of proximity and coordination should make mate guarding easy and effective enough to prevent EPC.

The opportunities for EPC are likely limited, too. The home ranges of our study groups have very little overlap (1.4\% on average (0-4.7), unpublished data; Fig. 1), and to find extra-pair mates, individuals would need to intrude into the neighboring home ranges, risking aggression from the same-sex residents. Another way to obtain EPC could be mating with floaters, solitary non-territorial individuals ranging over a wide area after having dispersed from their natal groups. There is accumulating evidence for the importance of floaters in population dynamics of both birds and mammals ${ }^{42,43}$. For example, in Azara's owl monkeys who are very similar to titis in all aspects of their social system, mated individuals experience intense intra-sexual competition from floaters of both sexes ${ }^{43,44}$. However, the evidence from Azara's owl monkeys and many other bird and mammal species indicate that floaters do not copulate with the mated animals as often as might be intuitively expected, and EPP are attributed to the neighboring individuals in most cases (e.g., Barelli et al., 2013; Nimje et al., 2019; Petrie \& Kempenaers, 1998; but see Cohas, Yoccoz, Da Silva, Goossens, \& Allainé, 2006; Kenyon, Roos, Binh, \& Chivers, 2011). In titis, only anecdotal reports of 
replacements by intruders exist ${ }^{49,50}$, but given the difficulty of detecting floaters, it is possible that they are present in titi populations, too. However, given the high levels of proximity and coordination between pair mates, EPC with the floaters are probably not easier to obtain than EPC with the neighboring individuals. Furthermore, EPC, whether with floaters or neighboring animals, might be costly, with the risks including the higher probability of acquiring sexually transmitted diseases and, for females, the retaliatory withholding of parental care by males ${ }^{51,52}$.

Opportunities for EPC are also affected by population density, with the higher densities making the encounters between individuals and, consequently, EPC less likely ${ }^{53}$. The positive relationship between population density and EPP rates was demonstrated, e.g., in Eurasian beavers, Castor fiber, and in many bird species $8,45,54,55$. Notably, the only published record of EPC in titis comes from a population of Plecturocebus ornatus (previously C. moloch) living in a disturbed habitat with exceptionally high density of 406 individuals $/ \mathrm{km}^{2}$ 56 . For comparison, the population density at our study site was estimated at 34 individuals $/ \mathrm{km}^{2}$ (unpublished data), and the average size of the home range of one group was 7.2 ha (Supplementary Table S1), bigger than the entire forest fragment of 6.9 ha inhabited by nine groups in the study of Mason (1966). The density of our study population was within the average range of values reported for undisturbed populations of titis 57-59; this relatively low density likely limited the opportunities for EPC. It should be mentioned, however, that for a population of Plecturocebus discolor from undisturbed habitat, a preliminary analysis reported three cases of EPP in a sample size of 16 offspring, although these data has not been published yet (Van Belle et al., 2016b, conference abstract). The density of this population (57 individuals $/ \mathrm{km}^{2}$ ) was higher than that of our study population, the home ranges were on average smaller (5.0 ha) and the percentage of home range overlap was larger (4.8\%) (Fernandez-Duque and Fiore, 2020), possibly accounting for the higher EPP rate.

Although in all cases of assigned paternities the social fathers were identified as genetic fathers for the group offspring (17 offspring born in 9 groups, up to 5 offspring generations per group), we cannot fully exclude the possibility of a low EPP rate in our study population. First, for one offspring (Group 10), paternity remained unassigned, as neither social father nor any other male from our sample was identified as the most likely father. While this case could be classified as neither extra- nor intra-pair paternity with confidence, it remains possible that this offspring was sired by an unsampled extra-pair male. In this case, the EPP rate in our study population would be $6 \%$. Alternatively (if we assume that the social father is indeed not the genetic father of the offspring), this case could be the result of a male replacement in a group. Adult replacements are known to happen in titis, with the breeding positions vacated after the disappearance (presumable deaths) of adults being occupied by same-sex immigrants ${ }^{33}$. Replacements can create groups that do not consist of biological parents and their offspring, leading to the apparent deviations from genetic monogamy even in the absence of EPC. As Group 10 was only habituated shortly before the genetic sample collection and no older offspring were present in it, we could not reconstruct its demographic history. Our data indicates that adult replacements do happen in our study population. The adult female of Group 4 was not identified as the genetic mother of the group's juvenile offspring, while the adult male was indicated as the genetic father. When we started following this group, the juvenile was estimated to be 7-8 months old based on its body size and the fact that it walked independently (juvenile titis start to walk on their own most of the time at the age of ca. 4.5 months: Jantschke et al., 1995). Lactation in titis lasts ca. 6.5 months ${ }^{62}$, and we did not see the female nursing. Therefore, we assume that the female replacement must have happened within ca. 2 months before we started following the groups, after the juvenile had been weaned.

Second, given the sample size of 17 offspring, maximum possible EPP level (assuming no EPP has been found and estimated with $95 \%$ confidence) will be $16.2 \%$, calculated as $\mathrm{y}=1-(1-\mathrm{x})^{17}$, where $\mathrm{y}$ is the probability of producing at least one extra-pair offspring (0.95); $\mathrm{x}$ is the frequency of EPP, and $\mathrm{n}$ is the sample size ${ }^{63}$. This confidence limit is a product of the sample size and does imply that there is $16.2 \%$ EPP rate in our study population. To narrow down the confidence interval to at least $5 \%$ of EPP, we would need a sample size of 58 offspring, which is difficult to achieve in a reasonable period in a secretive arboreal primate with slow life history, living in pairs and giving singleton births only once a year. 
Contrary to our predictions, we did not find evidence for relatedness- or heterozygosity-based mate choice in our study population. Interestingly, despite the absence of evidence for active inbreeding avoidance via mate choice, the pair mates in our study population were on average not related (mean $r=-0.033$ ) and never shared the same mtDNA haplotype (Supplementary Table S1, Fig. 2). Only in one case the pair mates were second-degree kin with $r=0.285$. Low relatedness between mating partners in the absence of active inbreeding avoidance was demonstrated in many other populations of mammals and birds, e.g., grey wolves, Canis lupus, arctic foxes, Vulpes lagopus, great reed warblers, Acrocephalus arundinaceus, and blue tits, Parus caeruleus ${ }^{24,64,65}$. In fact, active inbreeding avoidance via mate choice, although demonstrated in some birds and mammals (e.g., Hoffman et al., 2007; Leedale et al., 2020), was found in most pair-living species (García-Navas et al., 2009; Hansson et al., 2007; Schwensow et al., 2008; Sommer, 2005; reviewed in Jamieson et al., 2009). It has been suggested that in most situations, dispersal may be sufficient to avoid inbreeding ${ }^{24}$. By disrupting close-kin associations, dispersal can make the probability of encountering close kin relatively low, rendering active inbreeding avoidance via mate choice unnecessary ${ }^{66}$. In such cases, kin discrimination mechanisms might fail to evolve, and low inbreeding levels that will occasionally occur in such systems will be tolerated ${ }^{66}$.

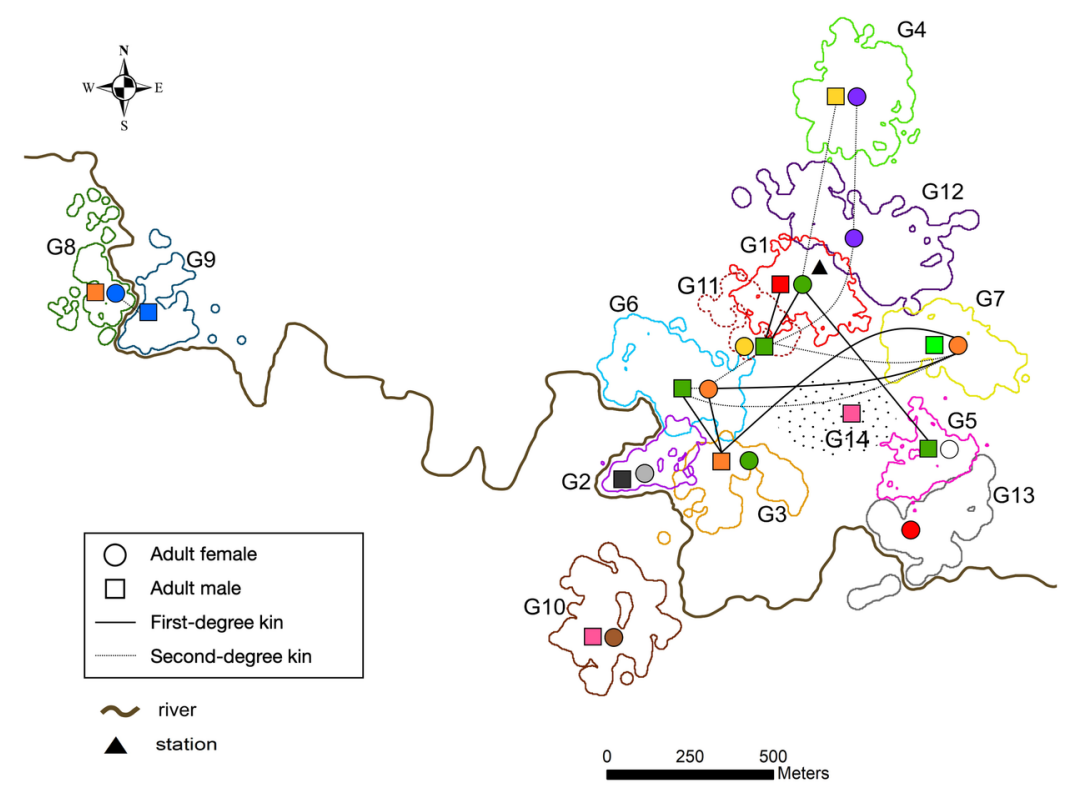

Figure 2: Home ranges, kinship and mtDNA haplotypes of adult females (circles) and males (squares) sampled in this study. The colors of circles and squares represent different mtDNA haplotypes

In our study population, dispersal was most likely opportunistic, as indicated by the absence of spatial genetic structure and the lack of geographic clustering in the mtDNA haplotype network (Fig. 2, 3). There was no obvious difference in dispersal distances between sexes, as both mtDNA haplotype diversity and mean relatedness were similar in females and males, suggesting that both sexes migrated over varying distances. As geographic scale of our study was confined, with the maximum distance between home-range centers of only $3200 \mathrm{~m}$, these results should be treated as preliminary. However, they are in line with the direct observation of dispersal and the kin structure of the study population. In one directly observed case of dispersal, a subadult male (the oldest offspring of Group 1), moved to an unoccupied area adjacent to his natal group and later formed a pair (Group 11) with an unknown female. The female did not have any first-degree kin among the sampled animals, indicating that she, unlike her mate, had not dispersed from any of the neighboring groups. The closest relative of this female was the adult male of Group 4 with $r=$ 0.156 (corresponding to a relatedness level between unrelated and half-sibling), with whom she also shared 
the same mtDNA haplotype (Supplementary Table S1). The kinship structure of the study population (Fig. 1) further suggested that while some dispersing individuals stay in the area (indicated by adult first-degree kin occupying home ranges that are either adjacent or separated by 1-2 home ranges), others migrate further (as many individuals in the study area are unrelated).

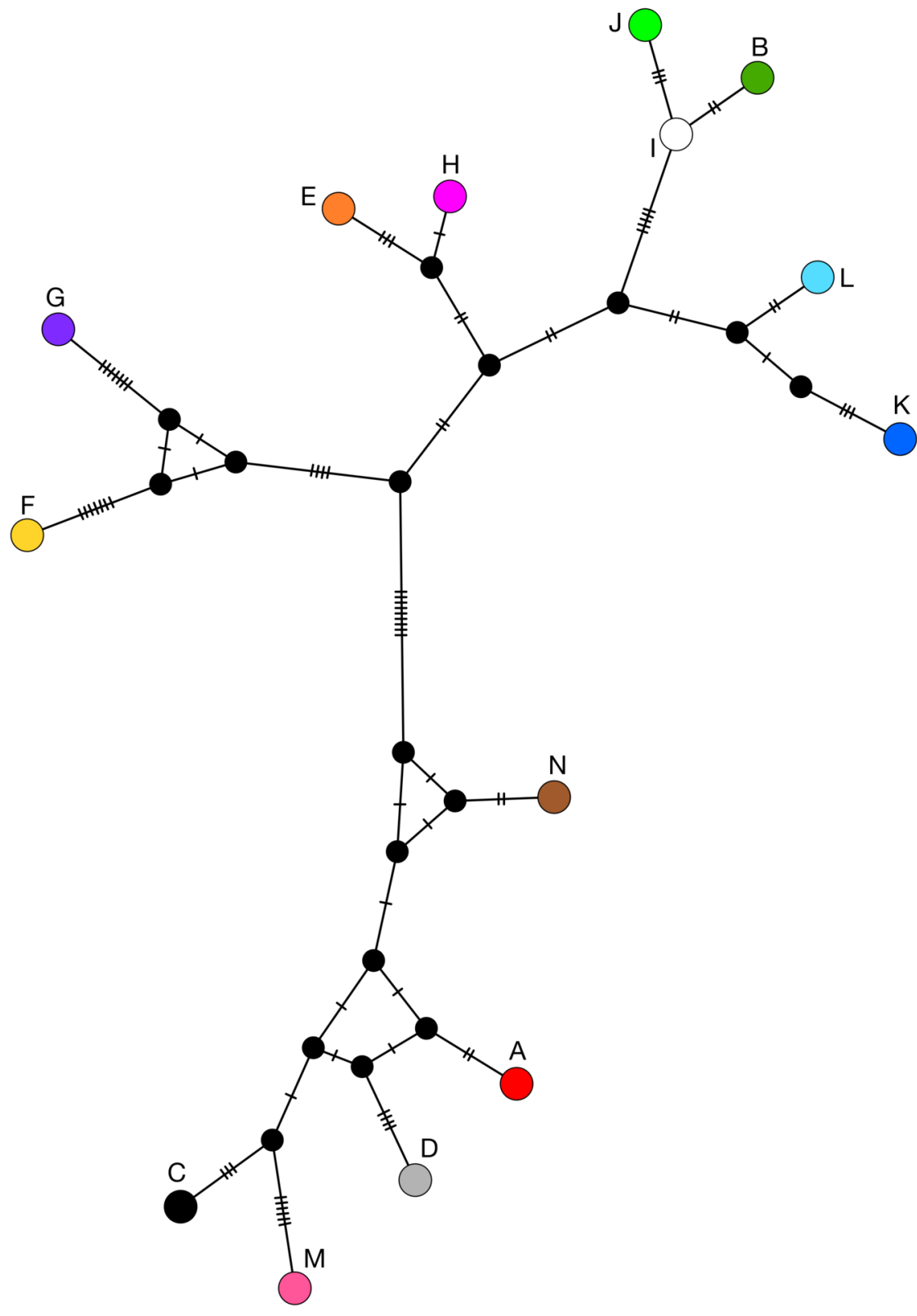

Figure 3: A median joining network of all mtDNA haplotypes found in our study groups, constructed in PopART ${ }^{67}$. The number of hatch marks indicates the number of mutations. Black nodes indicate inferred median vectors. The colors representing mtDNA haplotypes match those used in Fig. 1, 2. 
These results, although preliminary, are in line with the evidence from other studies indicating that even opportunistic dispersal can be sufficient to prevent inbreeding, as long as it is unconstrained by habitat fragmentation or other factors. The importance of unconstrained dispersal for inbreeding avoidance was supported in a simulation study based on empirical dataset from golden-crowned sifakas, Propithecus tattersalli, showing that high levels of outbreeding can be maintained in a population by a combination of social structure and unconstrained dispersal but without the need for active inbreeding avoidance mechanisms ${ }^{68}$. The link between dispersal and inbreeding risk was further indicated by studies demonstrating a correlation between dispersal distances and inbreeding level (e.g., in great tits, Parus major: Szulkin and Sheldon, 2008). At our study site, the habitat was undisturbed, and dispersal was most likely unconstrained, ensuring passive inbreeding avoidance. As indicated by one case where pair mates were second-degree kin, occasional inbreeding can still occur in such populations and is presumably tolerated.

In addition to dispersal and preferential mating with unrelated individuals, another potential way to avoid inbreeding is through engaging in EPC. Positive relationship between EPP rates and pair mate relatedness was demonstrated in many bird species ${ }^{7,10,11}$, but pair-living mammals do not seem to use this strategy often. In pair-living mammals, a similar strategy was, to our knowledge, only demonstrated in one species, fat-tailed dwarf lemur, where females sharing more MHC-supertypes with their social partner were shown to engaged in more EPC ${ }^{14}$. In our study population, the absence of evidence for EPP further confirms our suggestion that dispersal in this habitat is unconstrained and the potential for inbreeding is low, rendering EPC unnecessary.

Summing up, the current study is the first to examine the link between mating system, mate choice and dispersal in a wild population of a pair-living primate. We showed that coppery titis in our study population are mostly genetically monogamous, likely as a result of a strong pair bond enabling effective mate guarding and relatively low population density limiting the opportunities for extra-pair copulations. We further showed that coppery titis, despite exhibiting no active inbreeding avoidance via mate choice, still had low relatedness between pair mates. Our results indicate that even opportunistic dispersal, as long as it is not constrained, can create sufficient genetic dissimilarity between opposite sexes to render active mate choice and extra-pair copulations unnecessary. Future studies with larger sample size will be needed to examine the extent of genetic monogamy in different populations of coppery titis and to further investigate dispersal patters. In particular, to examine if titis indeed lack the mechanisms for active inbreeding avoidance via kin discrimination, it will be necessary to compare mating patterns and levels of inbreeding in continuous vs. fragmented or isolated populations. Finally, the absence of relatedness- and heterozygosity-based mate choice in our study population, of course, does not mean that mate choice does not occur in titis at any level. To better understand mating patterns in titis, future studies will have to examine if mate choice is based on other factors, such as, e.g., variation in MHC loci, body condition or the size or quality of the territory.

\section{Methods}

Study site and study population

The study was conducted at the Estacion Biologica Quebrada Blanco in the north-eastern Peruvian Amazon $\left(4^{\circ} 21^{\prime} \mathrm{S}, 73^{\circ} 09^{\prime} \mathrm{W}\right)$ in June 2017 - September 2019. Study individuals belonged to 14 family groups (Supplementary Table S1, Fig. 1), seven of which (Groups 1-7) were also subject to behavioral studies conducted in June-December 2017 and June-December 2018 38,70,71. Between the periods of behavioral data collection, the groups were monitored for 2-3 days per month, and genetic samples were collected continuously from the beginning of the study until September 2019. Group 1 had been habituated to the presence of human observers and studied intermittently since 1997; the other groups were habituated during this study. We individually identified all the study animals based on the combination of body size, tail shape and colouration, genitalia shape, and natural marks.

Titis typically give birth to a single infant once a year ${ }^{33,57,72}$. In our study population, most of the births occurred between October and February and only one occurred in June (Supplementary Table S1). As the offspring disperse at the age of $2-4$ years ${ }^{33,57,72}$, the pedigree in our study comprised up to 5 generations of 
offspring per group (Supplementary Table S1).

\section{Fecal sample collection and DNA extraction}

We collected fecal samples from 41 individuals (3-15 samples per individual) living in 14 family groups, including 18 putative offspring of 9 family groups ( 1 to 5 offspring per group). Five other groups either did not have offspring during the study period (or they had disappeared before we could collect samples) or the samples could not be collected because the offspring were still very young and thus their defecations too diminutive to be detected. Also due to differential habituation to the presence of humans, for some groups we could not obtain samples from all group members. For those groups that were habituated in the beginning of the study period, we collected samples from offspring from several consecutive years.

Fecal samples were collected immediately after an identified individual was seen defecating. We dried the samples in $15 \mathrm{~mL}$ falcon tubes containing silica gel beads (Carl Roth, Karlsruhe, Germany) and stored them at ambient temperature, replacing the silica beads when necessary, until shipping to Germany.

We extracted DNA (at least two samples per individual for all animals except one offspring of Group 10; see Results for more details) from ca. $200 \mathrm{mg}$ of feces using the Macherey-Nagel NucleSpinã DNA stool kit with a final elution of the DNA in $50 \mathrm{~mL}$ elution buffer. DNA concentration of the extracts was measured using a NanoDrop Spectrophotometer (ND-1000, PEQLAB Biotechnologie GmbH) and a Qubit Fluorometer (Thermo Fisher).

\section{Microsatellite genotyping}

As published microsatellite loci for titi monkeys ${ }^{73-75}$ revealed unreliable results for our study species, we established a new set of 27 di-repeat microsatellite loci that can be universally applied to New World monkeys (details are described in Supplementary Materials and Supplementary Tables S3-4). To simplify library preparation for genotyping by sequencing, we added adapter nucleotide sequences to the 5 ' end of the locus-specific primers.

We amplified all 27 loci in a single multiplex PCR using the Qiagen Multiplex PCR Kit (Qiagen) with a total volume of $25 \mathrm{~mL}$ and containing $12.5 \mathrm{~mL} 2 \mathrm{x}$ Multiplex Master Mix, $1 \mathrm{~mL}$ of primer pool $(0.2 \mathrm{mM}$ of each primer), $1 \mathrm{~mL}$ of DNA extract (ca. $200 \mathrm{ng}$ total DNA) and $10.5 \mathrm{~mL}$ of RNase-free water. Amplifications were performed with initial denaturation at $95^{\circ} \mathrm{C}$ for $15 \mathrm{~min}, 40$ cycles of denaturation at $94^{\circ} \mathrm{C}$ for 30 sec, annealing at $57{ }^{\circ} \mathrm{C}$ for $1.5 \mathrm{~min}$, extension at $72{ }^{\circ} \mathrm{C}$ for $1 \mathrm{~min}$ and a final extension at $60^{\circ} \mathrm{C}$ for $30 \mathrm{~min}$. PCR products were checked on $1.5 \%$ agarose gels together with non-template controls. To prevent false homozygosity due to allelic dropout, we repeated each multiplex reaction three times ${ }^{76}$. In some samples, the total multiplex reaction with all 27 loci yielded low number of sequencing reads; in these cases, we additionally amplified the loci in three separate multiplex reactions with the following primer pools: chr01b-chr07a, chr08a-chr12a, chr12b-chrXa, as this method usually yielded more reads (see Supplementary Materials for details). The reactions and PCR conditions for three separate multiplex reactions were the same as for the total multiplex reaction.

Following amplification, we pooled $5 \mathrm{~mL}$ of each multiplex PCR product (or of each PCR product of three separate multiplex reactions), purified the pooled products with the Monarch PCR \& DNA Cleanup Kit (New England BioLabs) and quantified them using Qubit Fluorometer (Thermo Fisher). To prepare sequencing libraries, we performed indexing PCRs using Kapa HiFi Hotstart ReadyMix PCR Kit (Roche) with a total volume of $25 \mathrm{~mL}$ containing $12.5 \mathrm{~mL}$ 2x Kapa HiFi Hotstart ReadyMix, $1 \mathrm{~mL}(0.5 \mathrm{mM})$ of each indexing primer (containing individual barcodes) and $100 \mathrm{ng}$ purified PCR product. Indexing PCRs were done with an initial denaturation step at $98^{\circ} \mathrm{C}$ for $45 \mathrm{sec}$, followed by 4 cycles of denaturation at $98^{\circ} \mathrm{C}$ for $15 \mathrm{sec}$, annealing at $62^{\circ} \mathrm{C}$ for $30 \mathrm{sec}$ and extension at $72{ }^{\circ} \mathrm{C}$ for $30 \mathrm{sec}$, and a final extension step at $72^{\circ} \mathrm{C}$ for 1 min. Full-length libraries were purified with the Monarch PCR \& DNA Cleanup Kit (New England BioLabs) and quantified using Qubit Fluorometer (Thermo Fisher). Fragment sizes and molarities were quantified using a Bioanalyzer 2100 (Agilent Technologies). Libraries were diluted to $10 \mathrm{nM}$ and then pooled and sequenced using Miseq Reagent Kit v2 with PhiX DNA (Illumina) added on the MiSeq system (Illumina). Sequencing 
was performed with 51 forward and 251 reverse read cycles. Only the reverse reads were used for further analysis, while forward reads were only used for MiSeq quality control.

To control for possible misidentification of animals in the field, we genotyped most individuals from 23 independent samples. We also used a PCR-based sexing assay 77 to confirm reported sex (and to sex young individuals for whom sex could not be identified in the field). To control for laboratory mistakes, we genotyped each sample twice, leading to at least four genotypes per individual.

After sequencing, the samples were demultiplexed using MiSeq Reporter software and then processed using the CHIIMP analysis pipeline ${ }^{76}$. The CHIIMP pipeline calls alleles by first producing unique sequences with relevant attributes (read counts, sequence length, etc.) for each MiSeq sequence file, querying the sequences for potential PCR artifacts, such as stutter sequences, and then removing all sequences that do not match the locus attributes. All alleles called by CHIIMP were manually checked to validate the results and to correct automated allele calling for those loci that contain "wobble" positions in the primer sequences and are incorrectly processed by CHIIMP. We used a cutoff of 250 reads. Additionally, we accepted alleles if they yielded $>100$ reads in at least three genotypes obtained per individual. Alleles with $<100$ reads were not called.

Of 27 loci, nine either consistently failed to amplify in our study animals (chr06b, chr11f, chr16b) or proved to be monomorphic (chr02a, chr02b, chr04a, chr10b, chr12a, chr13b) and were excluded from further analysis. The final set consisted of 18 loci, including 17 autosomal and one X-linked locus (chrXa) (Supplementary Table S5). All animals were genotyped at a minimum of 14 loci (16.8 loci on average), and the mean number of alleles per locus was 8.9.

We checked all loci for the presence of null alleles, allelic dropout and stuttering using Micro-Checker 2.2.5 78. We assessed Hardy-Weinberg equilibrium (HWE) and calculated observed and expected heterozygosity with PopGenReport 2.2.2 ${ }^{79}$. Since the presence of family structure can cause deviations from HWE and bias population genetic analyses, especially in monogamous species, we only included adults in this analysis. The analysis indicated that the population was in HWE. Two loci, chr01b and chr21a, departed from HWE, likely due to the presence of relatives in a study group and/or small sample size.

One of these two loci, chr01b, also showed evidence of null alleles. As the locus did not show any mismatches for the known mother/offspring dyads (see below), we ran all further analyses using two sets of data, one with the full set of loci and another one with locus chr01b excluded. Since the results from these two sets did not differ substantially, we present all further results only for the reduced data set.

\section{Mitochondrial DNA (mtDNA) genotyping}

We genotyped all individuals at the hypervariable region I of the mitochondrial control region using primers 5'-TACCTCGGTCTTGTAAACCG-3' and 5'-AGGTAGGAACCAGATGCCG-3', newly designed on the basis of mitochondrial genomes of New World monkeys available in GenBank. PCR reactions with a total volume of $30 \mu$ l contained $1 \mathrm{U}$ BiothermTaq 5000 (Genecraft), 1x reaction buffer, $0.16 \mathrm{mM}$ of each dNTP, 0.33 $\mu \mathrm{M}$ of each primer and ca. 100ng total DNA. PCRs were performed with initial denaturation at $95{ }^{\circ} \mathrm{C}$ for 2 min, 40 cycles of denaturation at $95^{\circ} \mathrm{C}$ for $1 \mathrm{~min}$, annealing at $58{ }^{\circ} \mathrm{C}$ for $1 \mathrm{~min}$, extension at $72{ }^{\circ} \mathrm{C}$ for $1 \mathrm{~min}$ and a final extension at $72{ }^{\circ} \mathrm{C}$ for $5 \mathrm{~min}$. PCR products were run on $1 \%$ agarose gels, excised from the gel and then purified with the Monarch DNA Gel Extraction Kit (New England BioLabs) and sequenced on an ABI 3130xL sequencer using both amplification primers and the BigDye Cycle Sequencing Kit (Applied Biosystems). Sequence electropherograms were checked with 4Peaks 1.8 (https://nucleobytes.com/4peaks/index.html) and manually edited and assembled in SeaView 4.5.4 ${ }^{80}$; all haplotypes were 567 bp long.

\section{Statistical analyses}

As X-linked loci are haploid in males and cannot be treated in the analyses in the same way as autosomal loci, all the following statistical tests were performed using the set of 16 autosomal loci for both sexes. The data for the X-linked locus chrXa was used separately to manually check for allelic mismatches between candidate parents and offspring in the parentage analyses. 


\section{(1) Parentage analyses}

Parentage was assigned using Cervus $3.0^{81}$. Cervus compares likelihood ratios of the two most likely candidate parents and assigns parentage based on statistical thresholds generated during the simulation analysis. For Cervus analysis, we used a simulation of 100,000 offspring, an error rate of $0.01,90 \%$ relaxed and $95 \%$ strict confidence level, and accounted for relatedness of candidate mothers and fathers. Relatedness was calculated with the $\mathrm{R}$ package related $1.0{ }^{82}$ using Wang's estimator $r{ }^{83}$. This estimator was chosen because it performed best in simulations, showing the highest correlation between observed and expected values for our set of loci. Additionally, we used Colony 2.0.6.5 ${ }^{84}$ to verify parentage assignments from Cervus. Unlike Cervus, Colony reconstructs a full pedigree, inferring sibship and parentage among individuals by comparing the likelihood of different clusters of individuals and maximizing group rather than pairwise likelihoods. For this analysis, we used an error rate of 0.01 , male and female polygyny, and a sibship size prior of 1.6, calculated as the average number of offspring per family group in our study population.

For both Cervus and Colony analyses, the set of candidate fathers included all sampled adult males plus the oldest subadult male from Group 6 that had dispersed from his natal group in the beginning of the study and could have sired offspring by the end of the sampling period. The set of candidate mothers included all adult females that shared their mtDNA haplotype with candidate offspring. For seven offspring (Supplementary Table S1), the mothers were known because they were seen nursing them. To test the reliability of our parentage estimates, we ran the analyses twice, with and without the respective set of known mother-offspring pairs. Combined non-exclusion probability for the set of 16 autosomal loci (with chr01b excluded) was $9.9 \times 10^{-5}$ for the first parent, $3.4 \times 10^{-7}$ for the second parent, and $9.0 \times 10^{-12}$ for the parent pair, calculated using Cervus.

\section{(2) Relatedness-based mate choice}

To test if titis avoid mating with related individuals, we compared relatedness between real and randomly generated mating partners using the pairwise relatedness estimator implemented in STORM ${ }^{85}$. First, STORM calculates the relatedness of real mating pairs using the estimator of Li et al. (1993), with each locus weighted using the method described in Lynch and Ritland (1999) and Van de Casteele et al. (2001). Then, the program calculates the expected relatedness of mating pairs if the mating is random with respect to relatedness; this is done by generating mating pairs from female and male breeding pools over 1000 iterations and averaging the relatedness values for each iteration. The obtained distribution is then compared to the averaged relatedness of real mating pairs. Our sample included ten real mating pairs, and the breeding pool consisted of 12 females and 12 males. This included all sampled adults and the oldest subadult male from Group 6.

\section{(3) Heterozygosity-based mate choice}

To test if titis show any heterozygosity-based mating pattern, we compared individual heterozygosity levels between pair mates. To estimate individual heterozygosity, we calculated homozygosity by loci (HL), a microsatellite-derived measure that weights the contribution of each locus to the homozygosity value depending on their allelic variability, implemented in R function GENHET $3.1^{89}$. To test if HL is correlated between pair mates, we used a two-tailed Pearson correlation analysis.

\section{(4) Dispersal patterns}

To examine if dispersal distances differ between sexes, we compared the diversity of mtDNA haplotypes, relatedness, and heterozygosity among adult females and males. MtDNA haplotype and nucleotide diversity was calculated and compared using a permutation test implemented in $\mathrm{R}$ function genetic_diversity_diff 1.0.6 (Alexander et al., 2016; available from https://github.com/laninsky/genetic_diversity_diffs). We included 12 sampled adult females and 12 adult males in this analysis, plus two females that could not be sampled but whose haplotypes were inferred from the haplotypes of their offspring (the adult female of Group 4, who supposedly had been replaced before the study period, and the adult female of Group 9, who was present during the study period but could not be sampled). Relatedness among females and among males 
was calculated using Wang's estimator $\mathrm{r}$ and then compared using 1000 bootstrapping samples in Coancestry 1.0.1.9 ${ }^{91}$. In this analysis, as well as in the tests described below, we included 12 sampled adult females and 12 males. Individual heterozygosity was calculated using HL estimator (homozygosity by locus, see above) and compared between sexes using a paired t-test.

To evaluate spatial genetic structure, we conducted a spatial autocorrelation analysis following Smouse and Peakall ${ }^{92}$ in PopGenReport 2.2.2 ${ }^{79}$, separately for adult females and males. The analysis calculated the correlation coefficient $r$ between pairwise genetic distances, calculated using microsatellite genotypes with the method of Smouse and Peakall ${ }^{92}$, and pairwise spatial distances, for each distance class. The coefficient $r$ is bound between -1 and 1 and has a mean of zero when there is no correlation. As a measure of spatial distances, we used distances between centroids of home ranges estimated using the $95 \%$ fixed kernel density method with ArcGIS Desktop 10.6 (ESRI). These distances varied from 215 to $3200 \mathrm{~m}$.

To further evaluate spatial genetic structure in females, we conducted a test similar to spatial autocorrelation analysis using mtDNA haplotype distances, correlating the number of nucleotide differences between haplotypes with spatial distances. For this test, if a spatial genetic structure is present, a positive correlation between haplotype and spatial distances is expected. We used Mantel tests with 10,000 permutations in $\mathrm{R}$ package ecodist ${ }^{93}$.

\section{(6) Kinship within and between groups}

Kinship was assigned conservatively based on $r$ values and pedigree reconstruction in Colony. First-degree kinship (full-sibling and parent-offspring, without distinguishing between these two categories) was assigned to dyads with $r>0.487$ (mean $r$ for simulated parent-offspring dyads). Second-degree kinship (dyads sharing $25 \%$ of the genome, such as half-sisters or uncle-nephew dyads) was assigned for dyads with $\mathrm{r}>0.247$ (mean $\mathrm{r}$ for simulated half-offspring dyads). Dyads with lower $\mathrm{r}$ values were categorized as unrelated.

\section{References}

1. Petrie, M. \& Kempenaers, B. Extra-pair paternity in birds: explaining variation between species and populations. Trends Ecol. Evol. 13, 52-58 (1998).

2. Clutton-Brock, T. Sexual selection in males and females. Science (80-. ). 318, 1882-1885 (2007).

3. Huck, M., Fernandez-Duque, E., Babb, P. L. \& Schurr, T. G. Correlates of genetic monogamy in socially monogamous mammals: insights from Azara's owl monkeys. Proc. R. Soc. B Biol. Sci. 281, 1-8 (2014).

4. Isvaran, K. \& Clutton-Brock, T. Ecological correlates of extra-group paternity in mammals. Proc. Biol. Sci. 274, 219-24 (2007).

5. Lukas, D. \& Clutton-Brock, T. H. The evolution of social monogamy in mammals. Science (80-. ). 341, 526-530 (2013).

6. Opie, C., Atkinson, Q. D., Dunbar, R. I. M. \& Shultz, S. Male infanticide leads to social monogamy in primates. Proc. Natl. Acad. Sci. U. S. A. 110, 13328-32 (2013).

7. Arct, A., Drobniak, S. M. \& Cichon, M. Genetic similarity between mates predicts extrapair paternity - a meta-analysis of bird studies. Behav. Ecol. 26, 959-968 (2015).

8. Brouwer, L. et al. Multiple hypotheses explain variation in extra-pair paternity at different levels in a single bird family. Molecular Ecology 26, (2017).

9. Trivers, R. L. Parental investment and sexual selection. in Sexual selection and the descent of man (ed. Campbell, B.) 136-179 (Aldine, 1972).

10. Jennions, M. D. \& Petrie, M. Why do females mate mulitply? A review of genetic benefits. Biol. Rev. 75, 21-64 (2000). 
11. Foerster, K., Delhey, K., Johnsen, A., Lifjeld, J. T. \& Kempenaers, B. Females increase offspring heterozygosity and fitness through extra-pair matings. Nature 425, 714-717 (2003).

12. Cohas, A. et al. The genetic similarity between pair members influences the frequency of extrapair paternity in alpine marmots. Anim. Behav. 76, 87-95 (2008).

13. Leclaire, S., Nielsen, J. F., Sharp, S. P. \& Clutton-Brock, T. H. Mating strategies in dominant meerkats: evidence for extra-pair paternity in relation to genetic relatedness between pair mates. J. Evol. Biol. 26, 1499-1507 (2013).

14. Schwensow, N., Fietz, J., Dausmann, K. \& Sommer, S. MHC-associated mating strategies and the importance of overall genetic diversity in an obligate pair-living primate. Evol. Ecol. 22, 617-636 (2008).

15. Kempenaers, B. Mate choice and genetic quality: a review of the heterozygosity theory. Adv. Study Behav. 37, 189-278 (2007).

16. Brown, J. L. A theory of mate choice based on heterozygosity. Behav. Ecol. 8, 60-65 (1997).

17. Zeh, J. A. \& Zeh, D. W. The evolution of polyandry II: post-copulatory defences against genetic incompatibility. Proc. R. Soc. B Biol. Sci. 264, 69-75 (1997).

18. Coltman, D., Pilkington, J., Smith, J. \& Pemberton, J. Parasite-mediated selection against inbred soay sheep in a free- living island population. Evolution (N. Y). 53, 1259-1267 (1999).

19. Ortego, J., Calabuig, G., Cordero, P. J. \& Aparicio, J. M. Egg production and individual genetic diversity in lesser kestrels. Mol. Ecol. 16, 2383-2392 (2007).

20. Hoffman, J. I., Forcada, J., Trathan, P. N. \& Amos, W. Female fur seals show active choice for males that are heterozygous and unrelated. Nature 445, 912-914 (2007).

21. García-Navas, V., Ortego, J. \& Sanz, J. J. Heterozygosity-based assortative mating in blue tits (Cyanistes caeruleus): implications for the evolution of mate choice. Proc. R. Soc. B Biol. Sci. 276, 29312940 (2009).

22. Sommer, S. Major histocompatibility complex and mate choice in a monogamous rodent. Behav. Ecol. Sociobiol. 58, 181-189 (2005).

23. Sin, Y. W. et al. MHC class II-assortative mate choice in European badgers (Meles meles). Mol. Ecol. 24, 3138-3150 (2015).

24. Hansson, B. et al. No evidence for inbreeding avoidance in a great reed warbler population. Behav. Ecol. 18, 157-164 (2007).

25. Leedale, A. E. et al. Cost, risk, and avoidance of inbreeding in a cooperatively breeding bird. Proc. Natl. Acad. Sci. 201918726 (2020). doi:10.1073/pnas.1918726117

26. Greenwood, P. J. Mating systems, philopatry and dispersal in birds and mammals. Anim. Behav. 28, 1140-1162 (1980).

27. Huchard, E., Knapp, L. A., Wang, J., Raymond, M. \& Cowlishaw, G. MHC, mate choice and heterozygote advantage in a wild social primate. Mol. Ecol. 19, 2545-2561 (2010).

28. Dobson, S. F. Competition for mates and predominant juvenile male dispersal in mammals. Anim. Behav. 30, 1183-1192 (1982).

29. Doolan, S. P. \& Macdonald, D. W. Dispersal and extra-territorial prospecting by slender-tailed meerkats (Suricata suricatta) in the south-western Kalahari. J. Zool. 240, 59-73 (1996).

30. Fernandez-Duque, E. Natal dispersal in monogamous owl monkeys (Aotus azarai) of the Argentinean Chaco. Behaviour 146, 583-606 (2009). 
31. Ribble, D. O. Dispersal in a monogamous rodent, Peromyscus californicus. Ecology 73, 859-866 (1992).

32. Favre, L., Balloux, F., Goudet, J. \& Perrin, N. Female-biased dispersal in the monogamous mammal Crocidura russula: evidence from field data and microsatellite patterns. Proc. R. Soc. B Biol. Sci. 264, 127-132 (1997).

33. Van Belle, S., Fernandez-Duque, E. \& Di Fiore, A. Demography and life history of wild red titi monkeys (Callicebus discolor) and equatorial sakis (Pithecia aequatorialis) in Amazonian Ecuador: a 12-year study. Am. J. Primatol. 78, 204-215 (2016).

34. Bicca-Marques, J. C. \& Heymann, E. W. Ecology and behavior of titi monkeys (genus Callicebus). in Evolutionary biology and conservation of titis, sakis and uacaris (eds. Barnett, A., Veiga, L. M., Ferrari, S. F. \& Norconk, M. A.) 196-207 (Cambridge University Press, 2013).

35. Anzenberger, G. The pairbond in the titi monkey (Callicebus moloch): intrinsic versus extrinsic contributions of the pairmates. Folia Primatol. 50, 188-203 (1988).

36. Fernandez-Duque, E., Fiore, A. Di \& de Luna, A. G. Pair-mate relationships and parenting in equatorial saki monkeys (Pithecia aequatorialis) and red titi monkeys $(</ \mathrm{i}>$ Callicebus discolor $</ \mathrm{i}>$ ) of Ecuador. in Evolutionary biology and conservation of titis, sakis and uacaris (eds. Veiga, L. M., Barnett, A. A., Ferrari, S. F. \& Norconk, M. A.) 295-302 (Cambridge University Press, 2013).

37. Oka, T. \& Takenaka, O. Wild gibbons' parentage tested by non-invasive DNA sampling and PCRamplified polymorphic microsatellites. Primates 42, 67-73 (2001).

38. Dolotovskaya, S., Walker, S. \& Heymann, E. W. What makes a pair bond in a Neotropical primate: female and male contributions. R. Soc. Open Sci. 7, 191489 (2019).

39. Kinzey, W. G. \& Wright, P. C. Grooming behavior in the titi monkey (Callicebus torquatus). Am. J. Primatol. 3, 267-275 (1982).

40. Spence-Aizenberg, A., Di Fiore, A. \& Fernandez-Duque, E. Social monogamy, male-female relationships, and biparental care in wild titi monkeys (Callicebus discolor). Primates 57, 103-112 (2016).

41. Kinzey, W. G. \& Robinson, J. G. Intergroup loud calls, range size, and spacing in Callicebus torquatus. Am. J. Phys. Anthropol. 60, 539-544 (1983).

42. Penteriani, V., Ferrer, M. \& Delgado, M. M. Floater strategies and dynamics in birds, and their importance in conservation biology: towards an understanding of nonbreeders in avian populations. Anim. Conserv. 14, 233-241 (2011).

43. Fernandez-Duque, E. \& Huck, M. Till death (or an intruder) do us part: intrasexual-competition in a monogamous primate. PLoS One 8, e53724 (2013).

44. Huck, M. \& Fernandez-Duque, E. Children of divorce: effects of adult replacements on previous offspring in Argentinean owl monkeys. Behav. Ecol. Sociobiol. 66, 505-517 (2012).

45. Nimje, P. S. et al. Almost faithful: SNP markers reveal low levels of extra-pair paternity in the Eurasian beavers. PeerJ Prepr. 7, e27866v1 (2019).

46. Barelli, C. et al. Extra-pair paternity confirmed in wild white-handed gibbons. Am. J. Primatol. 75, 1185-1195 (2013).

47. Cohas, A., Yoccoz, N. G., Da Silva, A., Goossens, B. \& Allainé, D. Extra-pair paternity in the monogamous alpine marmot (Marmota marmota): the roles of social setting and female mate choice. Behav. Ecol. Sociobiol. 59, 597-605 (2006). 
48. Kenyon, M., Roos, C., Binh, V. T. \& Chivers, D. Extrapair paternity in golden-cheeked gibbons (Nomascus gabriellae) in the secondary lowland forest of Cat Tien National Park, Vietnam. Folia Primatol. 82, 154-164 (2011).

49. Lawrence, J. Understanding the pair bond in brown titi monkeys (Callicebus brunneus): male and female reprodcutive interests (PhD thesis). PhD Thesis (Columbia University, New York, 2007).

50. Rodman, P. S. \& Bossuyt, F. J. Fathers and stepfathers: familial relations of old and new males within groups of Callicebus brunneus in southeastern Peru [Abstract]. Am J Phys Anthr. 132, 201 (2007).

51. Westneat, D. F. \& Stewart, I. R. K. Extra-pair paternity in birds: causes, correlates, and conflict. Annu. Rev. Ecol. Evol. Syst. 34, 365-396 (2003).

52. Poiani, A. \& Wilks, C. Sexually transmitted diseases: a possible cost of promiscuity in birds? Auk 117, 1061-1065 (2000).

53. Westneat, D. F. The ecology and evolution of extra-pair copulations in birds. Curr. Ornithol. 7, 331-369 (1990).

54. Westneat, D. F. \& Sherman, P. W. Density and extra-pair fertilizations in birds: a comparative analysis. Behav. Ecol. Sociobiol. 41, 205-215 (1997).

55. Syrůčková, A. et al. Genetic relationships within colonies suggest genetic monogamy in the Eurasian beaver (Castor fiber). Mammal Res. 60, 139-147 (2015).

56. Mason, W. A. Social organization of the South American monkey, Callicebus moloch: a preliminary report. Tulane Stud Zool 13, 23-28 (1966).

57. Bicca-Marques, J. C. \& Heymann, E. W. Ecology and behavior of titi monkeys (genus Callicebus). in Evolutionary biology and conservation of titis, sakis and uacaris (eds. Veiga, L. M., Barnett, A. A., Ferrari, S. F. \& Norconk, M. A.) 196-207 (Cambridge University Press, 2013).

58. Fernandez-Duque, E. \& Fiore, A. Di. The evolution of pair-living, sexual monogamy, and cooperative infant care: insights from research on wild owl monkeys, titi monkeys, sakis, and tamarins. Yearb. Phys. Anthropol. 1-86 (2020). doi:10.1111/j.1365-2958.2003.03935.x

59. Dacier, A., De Luna, A. G., Fernandez-Duque, E. \& Di Fiore, A. Estimating population density of Amazonian titi monkeys (Callicebus discolor) via playback point counts. Biotropica 43, 135-140 (2011).

60. Van Belle, S., Martins, A., Fernandez-Duque, E. \& Di Fiore, A. Patterns of paternity in wild socially monogamous titis (Callicebus discolor) and sakis (Pithecia aequatorialis) at the Tiputini Biodiversity Station, Ecuador (conference abstract). in International Primatological Society and American Society of Primatologists (2016).

61. Jantschke, B., Welker, C. \& Klaiber-Schuh, A. Notes on breeding of the titi monkey Callicebus cupreus. Folia Primatol. 65, 210-213 (1995).

62. Valeggia, C. R., Mendoza, S. P., Fernandez-Duque, E., Mason, W. A. \& Lasley, B. Reproductive biology of female titi monkeys (Callicebus moloch) in captivity. Am. J. Primatol. 47, 183-195 (1999).

63. Brotherton, P. N. M., Pemberton, J. M., Komers, P. E. \& Malarky, G. Genetic and behavioural evidence of monogamy in a mammal, Kirk's dik-dik (Madoqua kirkii). Proc. R. Soc. London B Biol. Sci. 264, 675-681 (1997).

64. Foerster, K., Valcu, M., Johnsen, A. \& Kempenaers, B. A spatial genetic structure and effects of relatedness on mate choice in a wild bird population. Mol. Ecol. 15, 4555-4567 (2006).

65. Geffen, E. et al. Kin encounter rate and inbreeding avoidance in canids. Mol. Ecol. 20, 5348-5358 (2011). 
66. Jamieson, I. G., Taylor, S. S., Tracy, L. N., Kokko, H. \& Armstrong, D. P. Why some species of birds do not avoid inbreeding: Insights from New Zealand robins and saddlebacks. Behav. Ecol. 20, 575-584 (2009).

67. Leigh, J. W. \& Bryant, D. POPART: Full-feature software for haplotype network construction. Methods Ecol. Evol. 6, 1110-1116 (2015).

68. Parreira, B., Quéméré, E., Vanpé, C., Carvalho, I. \& Chikhi, L. Genetic consequences of social structure in the golden-crowned sifaka. Heredity (Edinb). 1-12 (2020). doi:10.1038/s41437-020-0345-5

69. Szulkin, M. \& Sheldon, B. C. Dispersal as a means of inbreeding avoidance in a wild bird population. Proc. R. Soc. B Biol. Sci. 275, 703-711 (2008).

70. Dolotovskaya, S. \& Heymann, E. W. Do less or eat more: strategies to cope with costs of parental care in a pair-living monkey. Anim. Behav. 163, 163-173 (2020).

71. Dolotovskaya, S., Flores Amasifuen, C., Haas, C. E., Nummert, F. \& Heymann, E. W. Active antipredator behaviour of red titi monkeys (Plecturocebus cupreus). Primate Biol. 6, 59-64 (2019).

72. Souza-Alves, J. P., Caselli, C. B., Gestich, C. C. \& Nagy-Reis, M. B. Should I store, or should I sync? The breeding strategy of two small Neotropical primates under predictable resource availability. Primates 60, 113-118 (2019).

73. Mendoza, A. et al. Population genetics of the California National Primate Research Center's (CNPRC) captive Callicebus cupreus colony. Primates 56, 37-44 (2015).

74. Martins, A. B. Characterization and evaluation of microsatellite loci suitable for studies on mating system, parentage, and genetic identity in red titi monkeys (Callicebus discolor) and saki monkeys (Pithecia aequatorialis). (The University of Texas at Austin, 2015).

75. Menescal, L. A., Gonçalves, E. C., Silva, A., Ferrari, S. F. \& Schneider, M. P. C. Genetic diversity of red-bellied titis (Callicebus moloch) from Eastern Amazonia based on microsatellite markers. Biochem. Genet. 47, 235-240 (2009).

76. Barbian, H. J. et al. CHIIMP: an automated high-throughput microsatellite genotyping platform reveals greater allelic diversity in wild chimpanzees. Ecol. Evol. 16, 7946-7963 (2018).

77. Di Fiore, A. A rapid genetic method for sex assignment in non-human primates. Conserv. Genet. 6, 1053-1058 (2005).

78. Van Oosterhout, C., Hutchinson, W. F., Wills, D. P. M. \& Shipley, P. MICRO-CHECKER: software for identifying and correcting genotyping errors in microsatellite data. Mol. Ecol. Notes 4, 535-538 (2004).

79. Adamack, A. T. \& Gruber, B. PopGenReport: simplifying basic population genetic analyses in R. Methods Ecol. Evol. 5, 384-387 (2014).

80. Gouy, M., Guindon, S. \& Gascuel, O. Sea view version 4: a multiplatform graphical user interface for sequence alignment and phylogenetic tree building. Mol. Biol. Evol. 27, 221-224 (2010).

81. Kalinowski, S. T., Taper, M. L. \& Marshall, T. C. Revising how the computer program CERVUS accommodates genotyping error increases success in paternity assignment. Mol. Ecol. 16, 1099-1106 (2007).

82. Pew, J., Muir, P. H., Wang, J. \& Frasier, T. R. related: an R package for analysing pairwise relatedness from codominant molecular markers. Mol. Ecol. Resour. 15, 557-561 (2015).

83. Wang, J. An estimator for pairwise relatedness using molecular markers. Genetics 160, 1203-1215 (2002).

84. Jones, O. R. \& Wang, J. COLONY: a program for parentage and sibship inference from multilocus genotype data. Mol. Ecol. Resour. 10, 551-555 (2010). 
85. Frasier, T. R. STORM: software for testing hypotheses of relatedness and mating patterns. Mol. Ecol. Resour. 8, 1263-1266 (2008).

86. Li, C. C., Weeks, D. E. \& Chakravarti, A. Similarity of DNA fingerprints due to chance and relatedness. Hum. Hered. 43, 45-52 (1993).

87. Lynch, M. \& Ritland, K. Estimation of pairwise relatedness with molecular markers. Genetics 152, 1753-1766 (1999).

88. Van de Casteele, T., Galbusera, P. \& Matthysen, E. A comparison of microsatellite-based pairwise relatedness estimators. Mol. Ecol. 10, 1539-1549 (2001).

89. Coulon, A. Genhet: an easy-to-use R function to estimate individual heterozygosity. Mol. Ecol. Resour. 10, 167-169 (2010).

90. Alexander, A. et al. What influences the worldwide genetic structure of sperm whales (Physeter macrocephalus)? Mol. Ecol. 25, 2754-2772 (2016).

91. Wang, J. Coancestry: a program for simulating, estimating and analysing relatedness and inbreeding coefficients. Mol. Ecol. Resour. 11, 141-145 (2011).

92. Smouse, P. E. \& Peakall, R. Spatial autocorrelation analysis of individual multiallele and multilocus genetic structure. Heredity (Edinb). 82, 561-573 (1999).

93. Goslee, S. C. \& Urban, D. L. The ecodist package for dissimilarity-based analysis of ecological data. J. Stat. Softw. 22, 1-19 (2007).

94. Huck, M., Di Fiore, A. \& Fernandez-Duque, E. Of apples and oranges? The evolution of 'monogamy' in non-human primates. Front. Ecol. Evol. 7, 472 (2020).

95. Kappeler, P. M. A framework for studying social complexity. Behav. Ecol. Sociobiol. 73, 13 (2019).

96. Hennessy, C. A., Dubach, J. \& Gehrt, S. D. Long-term pair bonding and genetic evidence for monogamy among urban coyotes (Canis latrans). J. Mammal. 93, 732-742 (2012).

97. Ribble, D. O. The monogamous mating system of Peromyscus californicus as revealed by DNA fingerprinting. Behav. Ecol. Sociobiol. 29, 161-166 (1991).

98. Sommer, S. \& Tichy, H. Major histocompatibility complex (MHC) class II polymorphism and paternity in the monogamous Hypogeomys antimena, the endangered, largest endemic Malagasy rodent. Mol. Ecol. 8, 1259-1272 (1999).

99. Wu, J. S., Chiang, P. J. \& Lin, L. K. Monogamous system in the Taiwan vole Microtus kikuchii inferred from microsatellite DNA and home ranges. Zool. Stud. 51, 204-212 (2012).

100. Bonadonna, G. et al. Evidence of genetic monogamy in the lemur Indri (Indri indri). Am. J. Primatol. 81, e22993 (2019).

\section{Acknowledgments}

We thank Camilo Flores Amasifuén, Migdonio Huanuiri Arirama, Ney Shahuano Tello, Mathieu Maréchal, Sarah Walker, and all other field assistants without whom the field work would not have been possible. We also thank Christiane Schwarz for her excellent work in the genetics laboratory.

This work was conducted under all necessary permissions and ethical guidelines from the relevant authorities of Peru (research permit no. 249-2017-SERFOR/DGGSPFFS from the Servicio Nacional Forestal y de Fauna Silvestre of the Peruvian Ministry of Agriculture) and the German Primate Center.

The research was funded by German Primate Center, Leakey Foundation, German Research Foundation (DFG; Project nr. 407493972), International Primatological Society and Primate Action Fund. 


\section{Author contributions}

S.D., C.R. and E.W.H. designed the study. S.D. collected data in the field, did laboratory work and analysed the data. The paper was written by S.D., C.R. and E.W.H.

\section{Additional information}

Data Accessibility: All data needed to evaluate the conclusions in the paper, including genotypes of all study animals, are present in the paper and/or the Supplementary Materials. Additional data related to this paper may be requested from S.D. (s.dolotovskaya@gmail.com) or C.R. (croos@dpz.eu).

Competing Interests: The authors declare no competing interests.

Ethics: This work was conducted under all necessary permits (research permit no. 249-2017SERFOR/DGGSPFFS from the Servicio Nacional Forestal y de Fauna Silvestre of the Peruvian Ministry of Agriculture) and ethical guidelines from the relevant authorities of Peru and the German Primate Center.

\section{Tables}

Table 1. Terminology used in this study, proposed in (Huck, Di Fiore, \& Fernandez-Duque, 2020) and based on the framework of Kappeler (Kappeler, 2019).

Pair-living (who lives with whom): a type of social organization where one adult male and one adult female share a home range, possible with their non-reproducing offspring. This is often referred to as "social monogamy".

Pair-bonded (who is affiliated with whom): a type of social structure where one adult male and one adult female have an affiliative relationship to the exclusion of other adults, as evidenced by behavioral, emotional, and endocrinological characteristics.

Sexual monogamy (who mates with whom): a type of social mating system where one adult male and one adult female have an exclusive mating relationship during at least one reproductive season.

Genetic monogamy (who produces offspring with whom): a type of genetic mating system where one adult male and one adult female produce offspring exclusively with each other over a set of multiple births (at least one reproductive season for species that produce more than one infant per litter and over more than one consecutive reproductive seasons for species with singleton births)

Biparental care (who provides parental care): a type of care system where a mother and putative father regularly provide offspring care.

Table 2. List of genetically monogamous mammals with no extra-pair paternities (EPP) detected. Also included two predominantly genetically monogamous species with the EPP rate $<0.1$.

\begin{tabular}{ll}
\hline Species & Sample size \\
\hline Azara's owl monkey (Aotus azarae) & 35 offspring of 29 family groups (128 animals in total) \\
Bornean gibbon (Hylobates muelleri) & 4 offspring of 4 family groups $(13$ animals in total) \\
Kirk's dik-dik (Madoqua kirkii) & 12 offspring of 11 family groups $(68$ animals in total) \\
Coyotes (Canis latrans) & 96 offspring if 18 family groups (236 animals in total) \\
California mouse (Peromyscus californicus) & 82 offspring of 22 complete groups, plus 17 offspring from incomplet \\
Malagasy giant jumping rat (Hypogeomys antimena) & 60 offspring of 28 family groups (139 animals in total) \\
Taiwan vole (Microtus kikuchii) & 31 offspring of 20 family groups \\
Eurasian beaver (Castor fiber) & (a) 18 offspring of 9 colonies plus 6 family groups with only adults ( \\
Indri (Indri indri) & 12 offspring of 7 family groups (26 animals in total) \\
Golden-cheeked gibbons (Nomascus gabriellae) & 10 offspring of 6 family groups (29 animals in total) \\
White-handed gibbons (Hylobates lar) & 41 offspring, 27 born in pair-living groups and 15 born in multi-male \\
\hline
\end{tabular}

